\title{
ABSOLUTE LOCALIZATION OF CORNER REFLECTORS WITH TERRASAR-X SPOTLIGHT IMAGES
}

\author{
Ruiqing Song ${ }^{1 *}$, Jicang $\mathrm{Wu}^{1}$, Tao $\mathrm{Li}^{2}$, Fengming $\mathrm{Hu}^{1}$ \\ ${ }^{1}$ College of Surveying and Geo-Informatics, Tongji University, 200092 Shanghai, China - r.q.song@outlook.com \\ ${ }^{2}$ GNSS Research Center, Wuhan University, 430079 Wuhan, China - taoli@whu.edu.cn
}

KEY WORDS: Synthetic Aperture Radar, Absolute Localization, TerraSAR-X, Corner Reflector, Accuracy Analysis, Height Constraint

\begin{abstract}
:
Synthetic aperture radar, capable of imaging the Earth surface from space in nearly all-weather conditions and high spatial resolution, has shown its outstanding capability for a variety of ground mapping applications. With well-controlled orbits of the new generation SAR satellites, high accuracy absolute localization with multiple SAR images has been demonstrated and become one of the hot spots with increasing attention. In this paper, high-resolution Spotlight-mode TerraSAR-X images acquired from the single orbit track were applied to 3D absolute positioning of three triangular trihedral corner reflectors. In order to overcome the limitation imposed by the acquisitions with very short baselines, a height constraint was introduced and the sub-meter accuracy was derived after carefully compensating for the known error sources, such as atmospheric delays and solid earth tide shifts.
\end{abstract}

\section{INTRODUCTION}

Space-borne synthetic aperture radar (SAR), independent from weather and daytime, is recognized as an effective remote sensing technique for various Earth observation applications. By utilizing the phase differences between two SAR images acquired in same area with similar looking angle, SAR interferometry is capable of large continuous mapping and deformation monitoring of the Earth surface with centimeter to millimeter level accuracy. Besides, SAR also has geolocation ability of each image pixel based on the Earth elliptical model and Range-Doppler (R-D) equations (Curlander, 1982), but the location accuracy can barely reach meter-level for many years. With the improvement of the orbit and ranging accuracy of the recent generation SAR satellites, such as TerraSAR-X and Sentinel-1A/B, precise absolute localization with SAR images has gradually caused extensive concern. Since Eineder firstly introduced the concept of SAR imaging geodesy in 2011, many studies have demonstrated sub-meter level positioning accuracy of corner reflectors (CRs) with high resolution SAR images (Eineder et al., 2011; Cong et al., 2012; Balss et al., 2012; Balz et al., 2016). After carefully correcting the noise caused by atmospheric delays and Earth motion, the absolute geolocation in ITRF reference frame at centimeter-level can be obtained in case of TerraSAR-X (Schubert et al., 2010; Gisinger et al., 2015) and decimeter-level in case of Sentinel-1A/B (Schubert et al., 2017; Dheenathayalan et al., 2018). In this paper, we present our experiment of 3D absolute localization results with high-resolution Spotlight-mode TerraSAR-X images.

\section{METHODOLOGY}

As illustrated in figure 1, the basic idea of SAR absolute localization is geometrical intersection based on R-D equations by using slant range and precise satellite orbit information provided by multiple SAR images:

\footnotetext{
* Corresponding author
}

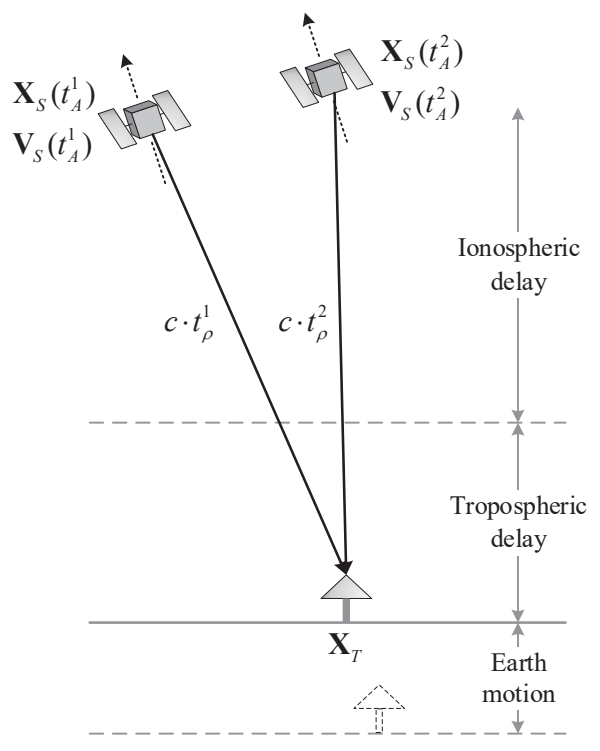

Figure 1. The typical geometry of SAR absolute localization.

$$
\begin{aligned}
& \left|\mathbf{X}_{S}-\mathbf{X}_{T}\right|-c \cdot t_{\rho}=0 \\
& \frac{\mathbf{V}_{S}\left(\mathbf{X}_{T}-\mathbf{X}_{S}\right)}{\left|\mathbf{V}_{S}\right|\left|\mathbf{X}_{S}-\mathbf{X}_{T}\right|}=\sin \alpha
\end{aligned}
$$

where $\mathbf{X}_{T}$ is the unknown target position vectors, $t_{\rho}$ indicates the one-way signal traveling time (range time) which measures distance between the sensor and the target by multiplying the velocity of light $c, \alpha$ is the squint angle which equals zero in case of SAR images corresponding to zero-Doppler geometry. $\mathbf{X}_{S}$ and $\mathbf{V}_{S}$ denote the sensor position and velocity vectors at the target imaging moment $t_{A}$ (azimuth time) respectively, and can be calculated by polynomial fitting from the discrete satellite state vectors provided in the SAR annotation files, and sixth order polynomials are enough for TerraSAR-X (Gisinger 
et al., 2015). Thus, as long as having two or more SAR images, the target position can be estimated by performing the least square adjustment iteratively after linearizing the observation equations. As there are two types of observations, variance component estimation should be implemented to determine appropriate weighting of each observation (Koch, Kusche, 2002).

To ensure the best possible location capability, two main challenges need to be considered:

(a) Homonymy point identification. The radar shadow and layover caused by SAR side looking geometric relations bring difficulty to identical point matching, especially for SAR images acquired from multiple sensor tracks. The triangle trihedral CRs have been wildly used as point targets in SAR image calibration and deformation monitoring for many years. Since it can reflects radar's incident wave three times back to the wave source, a well-designed CR can be presented as a bright target in SAR images, and its visibility is commonly measured by the signal-to-clutter ratio (SCR) (Freeman, 1992):

$$
S C R=\frac{\sigma_{C R} \sin \theta}{<\sigma_{0}>\rho_{r} \rho_{a}}
$$

where $\theta$ is local incident angle, $\rho_{r}$ and $\rho_{a}$ are the range and the azimuth resolution of SAR images, respectively. $\left\langle\sigma_{0}\right\rangle$ is the average radar background clutter power in slant range. $\sigma_{C R}$ is the radar cross section (RCS) value of CR. Under the ideal condition, the RCS of a triangle trihedral CR is given by (Ruck, 1970):

$$
\sigma_{C R}=\frac{4 \pi a^{4}}{3 \lambda^{2}}
$$

where $a$ is right-angle side length of a triangle trihedral CR, and $\lambda$ is radar wavelength. Actually, the RCS value decreases to some extent due to several factors, such as verticality among three reflecting surfaces and installation orientation. According to Freeman (1992), a CR can be easily identified when its SCR is better than $30 \mathrm{~dB}$, and then its approximate pixel position in each SAR image can be directly obtained. While each pixel is a synthesis of backscattered energy contributed by many distributed scatterers located within that pixel, the precise point target analysis needs to be implemented to measure the radar position $(r g, a z)$ with sub-pixel accuracy which is transformed to the range and the azimuth time observations:

$$
\begin{aligned}
& t_{\rho}=t_{\rho}^{0}+(r g-1) / r s r \\
& t_{A}=t_{A}^{0}+(a z-1) / p r f
\end{aligned}
$$

where $t_{\rho}^{0}$ and $t_{A}^{0}$ are time to the first range and azimuth pixel, $r s r$ is ranging sampling rate and $p r f$ is pulse repetition frequency. All of them can be read from SAR product annotation files.

(b) Observation corrections. Accurate timing system of SAR satellites is a crucial prerequisite for precise time observations according to equation (5). For TerraSAR-X, the so-called Instrument Fine Time (IFT) correction approach has been applied, and the timing system is designed to meet the requirements of sub-meter level localization (Balss et al., 2014). Meanwhile, as the conversion from range time to geometric distance depends

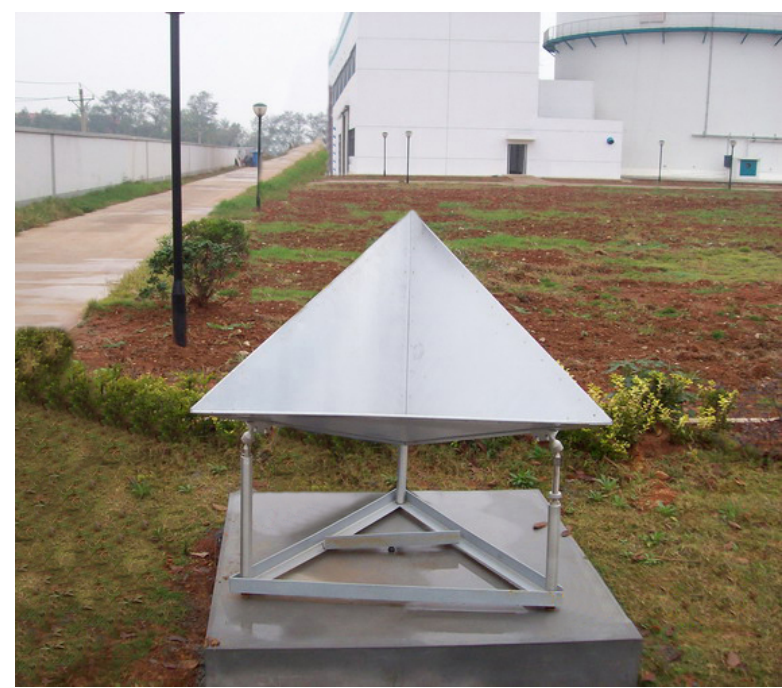

Figure 2. CR used in the experiment.

on the vacuum velocity of light, the range time should be corrected for signal propagation delays, mainly tropospheric delay and ionospheric delay. Besides, Earth motion will shift the true position of a ground target, such as earth tide and plate motion, should be taken into account.

Contrary to the SAR interferometry, the length of the spatial baseline should be as long as possible to ensure the geometrical stability of intersection positioning. Thus, it is a prior consideration to adopt SAR images acquired from different orbits or different satellite platforms. However, when only repeat pass SAR images from the same orbits with well-controlled spatial baselines can be obtained in the practical applications, it is difficult to acquire high precision positioning results. If the test area is flat and the precise height of ground targets are obtained from external high accuracy DSM, a height constraint can be introduced into localization calculation:

$$
(\cos B \cos L, \cos B \sin L, \sin B) \cdot(d x, d y, d z)^{T}=0
$$

where $B$ and $L$ are the rough initial latitude and longitude of the CRs location, $(d x, d y, d z)$ is the target location correction. Thus, the height of targets keep invariant during the iteration steps with this constraint, which assures the stability of positioning solutions.

\section{EXPERIMENTAL RESULTS}

Three triangular trihedral CRs with an edge length of $0.8 \mathrm{~m}$ were set up at State Grid Ultra High Voltage (UHV) test base, Wuhan, China, in 2008, as shown in figure 2. According to equation (4), the theoretical maximum RCS value of three CRs is $32.5 \mathrm{~dB} \cdot \mathrm{m}^{2}$. The ground coordinates of each CR's phase center were measured by GPS with centimeter-level accuracy. Three high-resolution Spotlight-mode (HS) TerraSAR-X SSC images of the same flight track were acquired. Table 1 lists the image parameters. The azimuth and range resolution of acquired images is respectively $0.84 \mathrm{~m}$ and $0.45 \mathrm{~m}$, which provides a very convenient condition for precise reflector peak returns location. 


\begin{tabular}{cccc}
\hline Date Mode & Heading $\begin{array}{c}\text { Incidence } \\
\text { angle }\left[{ }^{\circ}\right]\end{array}$ & $\begin{array}{c}\text { Resolution of } \\
\mathrm{Rg} / \mathrm{Az}[\mathrm{m}]\end{array}$ \\
\hline 2008/09/26 HS & Descending & 29.7 & $0.45 / 0.85$ \\
\hline 2008/10/07 HS & Descending & 29.7 & $0.45 / 0.85$ \\
\hline 2008/12/23 HS & Descending & 29.7 & $0.45 / 0.85$ \\
\hline
\end{tabular}

Table 1. Basic parameters of TerraSAR-X images used in this study.

Three CRs can be easily recognized in each SAR intensity image, as highlighted in red boxes in figure 3. Figure 4(a), (b) and (c) show the energy distribution features of each CR, respectively. The reflection power of three CRs is much higher than that in clutter areas, and has significant influence on adjacent eight pixels in red boxes. The SCR values of three CRs are $31.8 \mathrm{~dB}, 36.3 \mathrm{~dB}$ and $32.1 \mathrm{~dB}$, respectively. Following the idea presented in (Balss et al., 2012), the point target analysis was carried out for each CR by using a window size of $16 \times 16$ pixels centered at the corresponding CR. First, the cropped intensity map was oversampled by a factor of 32 using FFT based spectral zero-padding interpolation, and then the 2-D position in radar coordinates with sub-pixel accuracy was obtained by performing a polynomial refinement based on $3 \times 3$ pixels values around the intensity peak of oversampled image. Finally, the range and azimuth time observations of each CR were calculated according to equation (5).

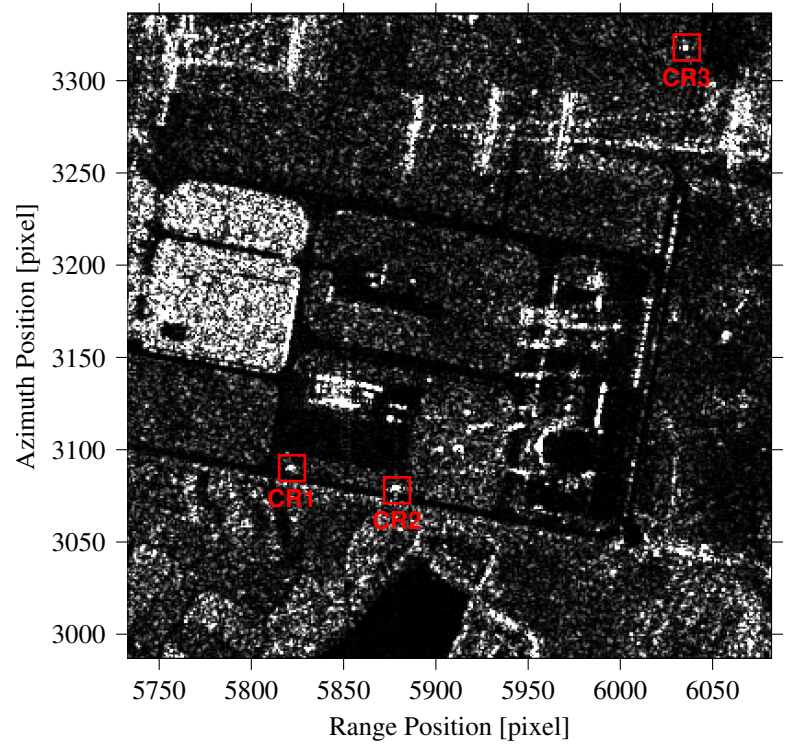

Figure 3. SAR average intensity map with three CRs highlighted in red box

The impact of the tropospheric delay (TD) and ionospheric delay (ID) on range time was corrected using GNSS atmospheric products provided by Crustal Dynamics Data Information System (CDDIS). For each CR, the vertical total electron content (VTEC) retrieved from TEC matrix was projected to slant range direction, and transformed to ID in meters by multiplying $40.28 / f^{2}$, where $f$ stands for the radar carrier frequency. The distribution of ionosphere is approximately between $100 \mathrm{~km}$ to $1000 \mathrm{~km}$, while the orbit altitude of TerraSAR-X is about $514 \mathrm{~km}$. Thus the ID correction was reduced by a scale of $75 \%$, and this value is obtained from

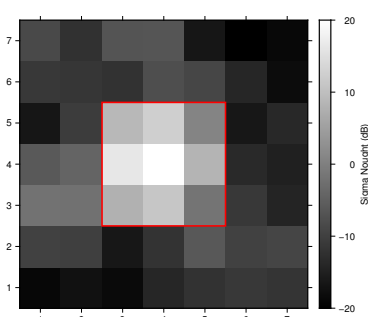

(a)

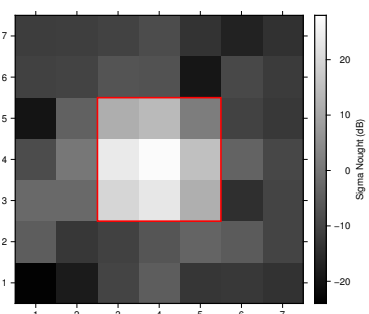

(b)

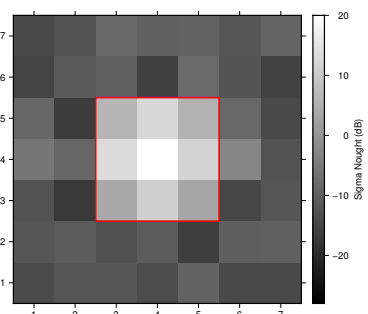

(c)
Figure 4. (a)/(b)/(c) Detailed energy distribution of CR1/CR2/CR3.

International Reference Ionosphere model (Bilitza, Reinisch, 2008). The TD of each CR was calculated by adopting the tropospheric zenith path delay (ZPD) provided by the IGS station WUH200CHN which is about $17 \mathrm{~km}$ away from CRs. Since an IGS station usually can cover a radius of $50 \mathrm{~km}$ for the troposphere, it is reasonable to select the above IGS station for TD correction.

The Earth motion caused by solid earth tide (SET) in the local North, East and Up frame of CR's ground location measured by GPS was computed by a program called solid.exe (available online: https://geodesyworld.github.io/SOFTS/solid.htm), and then was projected to slant range and azimuth direction. There is also azimuth shift (AS) which results from the relativistic Doppler effect or instrument timing errors and is annotated in the TerraSAR-X L1B products (Fritz et al., 2013). All observation corrections described above are listed in Table 2. It can be observed that the TD is the dominant effect and reaches several meters, while the ID is up to a few decimeters. The impact of the SET on range direction amounts from several centimeters to a few decimeters, and the amount on azimuth direction only varies at the millimeter level. The AS is a constant total shift and up to meters.

\begin{tabular}{cccccc}
\hline \multirow{2}{*}{ Date } & \multicolumn{3}{c}{ Rg Corr. } & \multicolumn{2}{c}{ Az Corr. } \\
\cline { 2 - 6 } & $\begin{array}{c}\text { SET } \\
{[\mathrm{m}]}\end{array}$ & $\begin{array}{c}\text { TD } \\
{[\mathrm{m}]}\end{array}$ & $\begin{array}{c}\text { ID } \\
{[\mathrm{m}]}\end{array}$ & $\begin{array}{c}\text { SET } \\
{[\mathrm{m}]}\end{array}$ & $\begin{array}{c}\text { AS } \\
{[\mathrm{m}]}\end{array}$ \\
\hline $09 / 26$ & 0.049 & 3.036 & 0.026 & -0.002 & -1.331 \\
\hline $10 / 07$ & -0.113 & 2.801 & 0.026 & 0.007 & -1.331 \\
\hline $12 / 23$ & 0.085 & 2.773 & 0.025 & 0.013 & -1.331 \\
\hline
\end{tabular}

Table 2. Observation corrections in meters.

According to the reference coordinates of CRs measured by GPS and satellite state vectors, the expected range and azimuth observations without any noises were calculated by searching the satellite positions satisfying the condition of zero-Doppler imaging geometry: (1) initialize the azimuth time at start and 


\begin{tabular}{ccccccccc}
\hline \multirow{2}{*}{ Site } & $\Delta X$ & $\Delta Y$ & $\Delta Z$ & $\sigma_{X}$ & $\sigma_{Y}$ & $\sigma_{Z}$ & $\sigma_{R}$ & $\sigma_{A}$ \\
\cline { 2 - 9 } & {$[\mathrm{m}]$} & {$[\mathrm{m}]$} & {$[\mathrm{m}]$} & {$[\mathrm{m}]$} & {$[\mathrm{m}]$} & {$[\mathrm{m}]$} & {$[\mathrm{m}]$} & {$[\mathrm{m}]$} \\
\hline CR1 & -0.1641 & 0.3127 & 0.5148 & 0.0600 & 0.0337 & 0.0438 & 0.0636 & 0.1048 \\
\hline CR2 & 0.5120 & -0.1349 & 0.4148 & 0.0414 & 0.0235 & 0.0307 & 0.0439 & 0.0735 \\
\hline CR3 & -0.9024 & -0.4363 & 0.4744 & 0.0601 & 0.0310 & 0.0382 & 0.0638 & 0.0907 \\
\hline
\end{tabular}

Table 4. Localization results of three CRs: difference between the estimated and the reference CR coordinates $(\Delta X, \Delta Y, \Delta Z)$, standard deviation of the estimated coordinates $\left(\sigma_{X}, \sigma_{Y}, \sigma_{Z}\right)$, standard deviation of range and azimuth observations $\left(\sigma_{R}, \sigma_{A}\right)$.

end of SAR image with 10 pixel margin, and calculate the corresponding satellite position and velocity; (2) calculate the projection of satellite velocity on the slant range direction according to the Doppler equation; (3) determine the CR's imaging azimuth time using Newton iteration method.

Table 3 reports the range and azimuth offsets between the expected and the corrected observations. Both the range and the azimuth offsets are at the decimeter-level after observation corrections. The standard deviations of range offsets amount within several centimeters, implying the range observations may contain unknown systematic errors to be calibration. In contrast, the standard deviations of azimuth offsets are up to decimeters, indicating that the TerraSAR-X timing procedure needs to be improved when aiming at centimeter-level localization accuracy.

\begin{tabular}{ccccccc}
\hline \multirow{2}{*}{ Date } & \multicolumn{2}{c}{$\mathrm{CR} 1[\mathrm{~m}]$} & \multicolumn{2}{c}{$\mathrm{CR} 2[\mathrm{~m}]$} & \multicolumn{2}{c}{$\mathrm{CR} 3[\mathrm{~m}]$} \\
\cline { 2 - 7 } & $\mathrm{Rg}$ & $\mathrm{Az}$ & $\mathrm{Rg}$ & $\mathrm{Az}$ & $\mathrm{Rg}$ & $\mathrm{Az}$ \\
\hline $09 / 26$ & 0.479 & 0.344 & 0.472 & 0.504 & 0.626 & 0.632 \\
\hline $10 / 07$ & 0.520 & 0.331 & 0.507 & 0.386 & 0.691 & 0.707 \\
\hline $12 / 23$ & 0.430 & 0.156 & 0.445 & 0.369 & 0.604 & 0.526 \\
\hline Stdev & 0.045 & 0.105 & 0.031 & 0.074 & 0.045 & 0.091 \\
\hline
\end{tabular}

Table 3. Range and azimuth offsets between the expected and the corrected observations.

However, these observations are acquired from the same flight track, and the maximum baseline is only about $600 \mathrm{~m}$, so the geometrical intersection is unreliable which will hamper the localization accuracy seriously. After adding the height constraint, the final localization result of each CR listed in Table 4 shows that the inner coincidence accuracy of target localization is all at centimeter-level, while the differences between the estimated and the reference CR coordinates are up to several decimeters. The average standard deviation of range is 0.057 and that of azimuth is 0.089 , which matches the observation offsets shown in table 3. Thus, the localization method performs well for SAR images of single orbit track after adding the height constraint.

\section{CONCLUDING REMARKS}

In this paper, the absolute localization of three triangle trihedral CRs with sub-meter accuracy is obtained by using highresolution Spotlight-mode TerraSAR-X images after range and azimuth corrections. In order to provide a reliable geometrical intersection, observations should be derived from SAR images of different orbit tracks. However, when only images of single orbit track are available or ground targets are only identified across images from the same orbit track, feasible localization solutions can still be obtained by introducing the proposed height constraint

\section{ACKNOWLEDGEMENTS}

This work is jointly supported by the Natural Science Foundation of China (Grant No. 41674003) and the DAAD TN 20192020 (Project No. 57421148).

\section{REFERENCES}

Balss, U., Breit, H., Fritz, T., Steinbrecher, U., Gisinger, C., Eineder, M., 2014. Analysis of internal timings and clock rates of TerraSAR-X. in Proc. IEEE IGARSS Conf., 2671-2674.

Balss, U., Cong, X. Y., Brcic, R., Rexer, M., Minet, C., Breit, H., Eineder, M., Fritz, T., 2012. High precision measurement on the absolute localization accuracy of TerraSAR-X. in Proc. IEEE IGARSS Conf., 1625-1628.

Balz, T., Wang, J. H., Liao, M. S., 2016. Absolute geopositioning accuracy of TerraSAR-X-Experimental validation in Wuhan. in Proc. IEEE IGARSS Conf., 1086-1089.

Bilitza, D., Reinisch, B., 2008. International reference ionosphere 2007: Improvements and new parameters. Adv. Space Res., 42(4), 599-609.

Cong, X. Y., Balss, U., Eineder, M., Fritz, T., 2012. Imaging geodesy-Centimeter-level ranging accuracy with TerraSARX: An update. IEEE Geosci. Remote Sens. Lett., 9(5), 948-952.

Curlander, J. C., 1982. Location of spaceborne SAR imagery. IEEE Trans. Geosci. Remote Sens., GE-20(3), 359-364.

Dheenathayalan, P., Small, D., Hanssen, R. F., 2018. 3-D positioning and target association for medium-resolution SAR sensors. IEEE Trans. Geosci. Remote Sens., 56(11), 68416853.

Eineder, M., Minet, C., Steigenberger, P., Cong, X. Y., Fritz, T., 2011. Imaging geodesy-Toward centimeter-level ranging accuracy with TerraSAR-X. IEEE Trans. Geosci. Remote Sens., 49(2), 661-671.

Freeman, A., 1992. SAR calibration: An overview. IEEE Trans. Geosci. Remote Sens., 30(6), 1107-1121.

Fritz, T., Eineder, M., Mittermayer, J., Schättler, B., Balzer, W., Buckreuß, S., Werninghaus, R., 2013. TerraSAR-X ground segment basic product specification document. TX-GS-DD-3302, v1.9. Available: http://terrasar-x.dlr.de/. 
Gisinger, C., Balss, U., Pail, R., Zhu, X. X., Montazeri, S., Gernhardt, S., Eineder, M., 2015. Precise three-dimensional stereo localization of corner reflectors and persistent scatterers with TerraSAR-X. IEEE Trans. Geosci. Remote Sens., 53(4), 1782-1802.

Koch, K. R., Kusche, J., 2002. Regularization of geopotential determination from satellite data by variance components. $J$. Geod., 76(5), 259-268.

Ruck, G. T., 1970. Radar cross section handbook. Plenum Publishing Corporation.

Schubert, A., Jehle, M., Small, D., Meier, E., 2010. Influence of atmospheric path delay on the absolute geolocation accuracy of TerraSAR-X high-resolution products. IEEE Trans. Geosci. Remote Sens., 48(2), 751-758.

Schubert, A., Miranda, N., Geudtner, D., Small, D., 2017. Sentinel-1A/B combined product geolocation accuracy. Remote Sens., 9(6), 607. 